\title{
TRANSFER OF A UNIT OF GOOD BETWEEN AGENTS IN CASES OF DEGRESSIVELY PROPORTIONAL ALLOCATION
}

\author{
Janusz Łyko
}

\begin{abstract}
The paper discusses the conditions of transferring one unit of a good between agents so as to satisfy the principle of degressively proportional allocation. Solving this problem is a prerequisite for possible modifications of some proportional division methods if we want to apply them in the degressively proportional allocation of goods. In particular, these methods use the relation of priority and are based on the minimization of certain measures of disproportionateness. It turns out that the well-established approaches to proportional allocation are not always possible. The research findings presented in the paper demonstrate that the strongest constraint in this problem is the potential small diversification of the sequence of claims of individual agents.
\end{abstract}

Keywords: degressive proportionality, fair division, European Parliament, voting.

JEL Classification: D39, D63.

DOI: $10.15611 /$ me.2016.12.03.

\section{Introduction}

A proportional division of goods, generally accepted as fair in the European culture, does not always lead to a generally accepted solution. There are situations where agents agree to deviate from classical rules for the sake of reaching a common, desired goal, such as the case of distributing seats in the European Parliament among the member states. As a result of considerable differences in the population numbers of the member states, the Treaty of Lisbon [The Treaty of Lisbon 2007] stipulated degressive proportionality as the rule of the allocation of parliamentary mandates. The rule enables all member states to be justly represented while keeping a the entire assembly at a reasonable size. Article 9A of the Lisbon Treaty reads: "The European Parliament shall be composed of representatives of the Union's citizens. They

\footnotetext{
Janusz Lyko

Department of Mathematics and Cybernetics, Wrocław University of Economics

janusz.lyko@ue.wroc.pl
} 
shall not exceed seven hundred and fifty in number, plus the President. Representation of citizens shall be degressively proportional, with a minimum threshold of six members per Member State. No Member State shall be allocated more than ninety-six seats".

A precise statement of this provision is given by the 2007 resolution of the European Parliament: "the larger the population of a country, the greater its entitlement to a large number of seats", and "the larger the population of a country, the more inhabitants are represented by each of its Members of the European Parliament” [Lamassoure, Severin 2007]. In addition to the rule itself, this article also indicates the so-called boundary conditions of allocation, i.e. the minimum number of seats allocated to the smallest country, and the maximum number of seats allocated to the greatest country. The original constraints were given as inequalities, however following the current interpretation we assume they specify the exact numbers of seats allocated to Malta and to Germany: $s_{1}=m=6$ and $s_{n}=M=96$. Also the total size $H$ of allocated good is often treated as a boundary condition [Łyko 2012] and [Dniestrzański, Łyko 2014]. In our case it is the total number of seats, equal to 751 .

We reject the concept of unrounded degressive proportionality known in the literature [Delgado-Márquez et al. 2013], which assumes that the degressive proportionality condition should hold only before rounding to integers, and we formally define the set of feasible solutions to the problem of degressively proportional, integer allocation of $H$ units of a good among $n$ agents, whose claims are defined by the sequence $P=\left(p_{1}, p_{2}, \ldots, p_{n}\right)$, where $0<p_{1} \leq p_{2} \leq \ldots \leq p_{n}$, and satisfying given boundary conditions $s_{1}=m$ and $s_{n}=M$. The set $D P(P, H, m, M)$ is the set of all sequences $S=\left(s_{1}, s_{2}, \ldots, s_{n}\right)$ of integer terms such that:

(1) $s_{i} \leq s_{i+1}$ for each $1 \leq i<n$,

(2) $\frac{p_{i}}{s_{i}} \leq \frac{p_{i+1}}{s_{i+1}}$ for each $1 \leq i<n$,

(3) $s_{1}=m$ and $s_{n}=M$,

(4) $s_{1}+s_{2}+\cdots+s_{n}=H$.

\section{Problem formulation}

The theory of the fair distribution of indivisible goods includes the problems of allocation where a given relation generates priorities or claims to obtain a good. Other problems are also studied that assume minimization of 
a certain measure of disproportionateness [Pukelsheim 2013]. In both cases the concept of transferring a unit of good from one agent to another is useful. Then a relocation means that first, a good is moved from a less entitled agent to a more entitled agent, and second, the measure of disproportionateness is lessened. There are no additional constraints for executed transfers, because each solution is feasible. We deal with a different situation when transfers are executed in degressively proportional allocations. In this case the condition of degressive proportionality must also be satisfied by the sequence generated after the transfer, i.e. this sequence must be an element of the set $D P(P, H, m, M)$. Therefore, if we try to modify such methods in order to employ them in the case of degressively proportional allocation, we have to begin by determining when a transfer of one unit of good between agents does not lead to the violation of the principle of degressive proportionality.

Let us assume that given $P, H, m, M$, the set $D P(P, H, m, M)$ is not empty and that $S=\left(s_{1}, s_{2}, \ldots, s_{n}\right)$ is an element of this set.

Let

$$
\mathrm{S}_{\mathrm{k}, \mathrm{l}}^{+}=\left(\mathrm{s}_{1}, \ldots, \mathrm{s}_{\mathrm{k}-1}, \mathrm{~s}_{\mathrm{k}}-1, \mathrm{~s}_{\mathrm{k}+1}, \ldots \mathrm{s}_{\mathrm{l}-1}, \mathrm{~s}_{\mathrm{l}}+1, \mathrm{~s}_{\mathrm{l}+1}, \ldots, \mathrm{s}_{\mathrm{n}}\right)=\left(\mathrm{s}_{1}^{+}, \mathrm{s}_{2}^{+}, \ldots, \mathrm{s}_{\mathrm{n}}^{+}\right)
$$

and

$$
S_{k, l}^{-}=\left(s_{1}, \ldots, s_{k-1}, s_{k}+1, s_{k+1}, \ldots, s_{l-1}, s_{l}-1, s_{l+1}, \ldots, s_{n}\right)=\left(s_{1}^{-}, s_{2}^{-}, \ldots, s_{n}^{-}\right)
$$

denote an allocation generated from $S$ after transferring one unit of the good from agent $k$ to agent $l$ or from $l$ to $k$, respectively. Since $s_{1}=m$ and $s_{n}=M$ have fixed values, one has to assume that $1<k<l<n$, which also implies that the problem can be considered only if $n>3$. Its solution consists in identifying such conditions that

$$
S_{k, l}^{+} \in D P(P, H, m, M) \text { and } S_{k, l}^{-} \in D P(P, H, m, M) .
$$

\section{Transfer of goods vs. degressive proportionality}

By definition of elements $S_{k, l}^{+}$and $S_{k, l}^{-}$, they meet condition (3) in the definition of the set $D P(P, H, m, M)$. It is easy to notice that both $S_{k, l}^{+}$and $S_{k, l}^{-}$ also meet condition (4). Transferring one unit of the good between agents does not affect the total $H$. Therefore it suffices to consider merely conditions (1) and (2).

For any $\mathrm{S} \in D P(P, H, m, M)$ and $1<i<n$, we have

$$
\frac{p_{i-1}}{s_{i-1}} \leq \frac{p_{i}}{s_{i}} \text { and thus } \frac{p_{i-1}}{s_{i-1}} \leq \frac{p_{i}}{s_{i}-1}
$$


and

$$
\frac{p_{i}}{s_{i}} \leq \frac{p_{i+1}}{s_{i+1}} \text { implying } \frac{p_{i}}{s_{i}+1} \leq \frac{p_{i+1}}{s_{i+1}} .
$$

As a result, we have $\frac{p_{k-1}}{s_{k-1}^{+}} \leq \frac{p_{k}}{s_{k}^{+}}$and $\frac{p_{l}}{s_{l}^{+}} \leq \frac{p_{l+1}}{s_{l+1}^{+}}$, and also

$$
\frac{p_{k}}{s_{k}^{-}} \leq \frac{p_{k+1}}{s_{k+1}^{-}} \text {and } \frac{p_{l-1}}{s_{l-1}^{-}} \leq \frac{p_{l}}{s_{l}^{-}} \text {for any } 1<k<l<n .
$$

The remaining inequalities, i.e.

$$
s_{k-1}^{+} \leq s_{k}^{+} \leq s_{k+1}^{+}, s_{l-1}^{+} \leq s_{l}^{+} \leq s_{l+1}^{+}, \frac{p_{k}}{s_{k}^{+}} \leq \frac{p_{k+1}}{s_{k+1}^{+}}, \frac{p_{l-1}}{s_{l-1}^{+}} \leq \frac{p_{l}}{s_{l}^{+}}
$$

and

$$
s_{k-1}^{-} \leq s_{k}^{-} \leq s_{k+1}^{-}, s_{l-1}^{-} \leq s_{l}^{-} \leq s_{l+1}^{-}, \frac{p_{k-1}}{s_{k-1}^{-}} \leq \frac{p_{k}}{s_{k}^{-}}, \frac{p_{l}}{s_{l}^{-}} \leq \frac{p_{l+1}}{s_{l+1}^{-}},
$$

do not necessarily hold in a general case. Diversification of elements of the sequence $P$ considerably affects whether they hold. To be more specific, extremely small differences between neighboring terms of the sequence, i.e. between $p_{i}$ and $p_{i+1}$, lead to such allocations, given the boundary conditions $H, m$ and $M$, that all the elements of the set $D P(P, H, m, M)$ satisfy the equality

$$
s_{i}=s_{i+1} .
$$

Example. Given the set $P=(100,201,403,405,750,100)$ with $m=4$, $M=20$ and $H=70$, the set $D P(P, H, m, M)$ contains six elements. It is easy to see that

$$
\begin{gathered}
D P(P, 70,4,20)=\{(4,6,11,11,18,20),(4,8,10,10,18,20),(4,7,11,11,17,20), \\
(4,6,12,12,16,20),(4,8,11,11,16,20),(4,7,12,12,15,20)\} .
\end{gathered}
$$

In addition, the allocation $S=(4,8,10,10,18,20)$ for any $1<k<l<6$ satisfies $S_{k, l}^{+} \notin D P(P, 70,4,20)$ and $S_{k, l}^{-} \notin D P(P, 70,4,20)$.

Proposition 1. If $\mathrm{S} \in D P(P, H, m, M), s_{k-1}<s_{k}$ and $s_{l}<s_{l+1}$ with $\frac{p_{k}}{p_{k+1}} \leq \frac{s_{k}}{s_{k+1}}-\frac{1}{s_{k+1}}$ and $\frac{p_{l}}{p_{l-1}} \geq \frac{s_{l}}{s_{l-1}}+\frac{1}{s_{l-1}}$, then $S_{k, l}^{+} \in D P(P, H, m, M)$.

Proof. It is known that $s_{k-1}<s_{k}$, hence

$$
s_{k-l}^{+}=s_{k-1} \leq s_{k}-1=s_{k}^{+}=s_{k} \leq s_{k+1}=s_{k+1}^{+} \text {. }
$$


In the same way, the inequality $s_{l}<s_{l+1}$ results in

$$
s_{l-1}^{+}=s_{l-1} \leq s_{l} \leq s_{l}+1=s_{l}^{+} \leq s_{l+1}=s_{l+1}^{+} \text {. }
$$

As a result, the condition (1) in the definition of the set $D P(P, H, m, M)$ is satisfied. In addition, if $\frac{p_{k}}{p_{k+1}} \leq \frac{s_{k}}{s_{k+1}}-\frac{1}{s_{k+1}}$, then

$$
\frac{p_{k}}{p_{k+1}} \leq \frac{s_{k}-1}{s_{k+1}} \text { and } \frac{p_{k}}{s_{k}-1}=\frac{p_{k+1}}{s_{k+1}} \text {, that implies } \frac{p_{k}}{s_{k}^{+}} \leq \frac{p_{k+1}}{s_{k+1}^{+}} \text {. }
$$

Likewise the inequality $\frac{p_{l}}{p_{l-1}} \geq \frac{s_{l}}{s_{l-1}}+\frac{1}{s_{l-1}}$ results in

$$
\frac{p_{l}}{p_{l-1}} \geq \frac{s_{l}+1}{s_{l-1}} \text { and } \frac{p_{l}}{s_{l}+1} \geq \frac{p_{l-1}}{s_{l-1}} \text {, thus implying that } \frac{p_{l-1}}{s_{l-1}^{+}} \leq \frac{p_{l}}{s_{l}^{+}} \text {. }
$$

In this way, one can also prove that proposition 2 is true.

Proposition 2. If $\mathrm{S} \in D P(P, H, m, M), s_{k}<s_{k+1}$ and $s_{l-1}<s_{l}$, with

$$
\frac{p_{k}}{p_{k-1}} \geq \frac{s_{k}}{s_{k-1}}+\frac{1}{s_{k-1}} \text { and } \frac{p_{l}}{p_{l+1}} \leq \frac{s_{l}}{s_{l}+1}-\frac{1}{s_{l}+1} \text {, then } S_{k, l}^{-} \in D P(P, H, m, M) \text {. }
$$

Proof. The condition $s_{k}<s_{k+1}$ results in

$$
s_{k-1}^{-}=s_{k-1} \leq s_{k} \leq s_{k}+1=s_{k}^{-} \leq s_{k+1}=s_{k+1}^{-} \text {, }
$$

whereas the inequality $s_{l-1}<s_{l}$ leads to

$$
s_{l-1=}^{-} s_{l-1} \leq s_{l}-1=s_{l}^{-} \leq s_{l+1}=s_{l+1}^{+} .
$$

In addition, if $\frac{p_{k}}{p_{k-1}} \geq \frac{s_{k}}{s_{k-1}}+\frac{1}{s_{k-1}}$, then

$$
\frac{p_{k}}{p_{k-1}} \geq \frac{s_{k}+1}{s_{k-1}} \text { and } \frac{p_{k}}{s_{k}+1} \geq \frac{p_{k-1}}{s_{k-1}} \text {, thus implying that } \frac{p_{k-1}}{s_{k-1}^{-}} \leq \frac{p_{k}}{s_{k}^{-}} \text {. }
$$

The inequality $\frac{p_{l}}{p_{l+1}} \leq \frac{s_{l}}{s_{l}+1}-\frac{1}{s_{l}+1}$ results in $\frac{p_{l}}{p_{l+1}} \leq \frac{s_{l}-1}{s_{l+1}}$ and $\frac{p_{l}}{s_{l}-1} \leq \frac{p_{l+1}}{s_{l+1}}$ that completes the proof, because it means that $\frac{p_{l}}{s_{l}^{-}} \leq \frac{p_{l+1}}{s_{l+1}^{-}}$.

Propositions 1 and 2 specify the conditions which must be satisfied if a transfer of a good between fixed entities $k$ and $l$ is required. The conditions are not general because specific values of the sequence $S$ are referred to. Therefore it is still unknown in general cases how to specify sequences $S$ which satisfy

$$
S_{k, l}^{+} \in D P(P, H, m, M) \text { and } S_{k, l}^{-} \in D P(P, H, m, M) .
$$


If the set $D P(P, H, m, M)$ is not empty, then a sequence

$$
S^{\text {min }}=\left(s_{1}^{\text {min }}, s_{2}^{\text {min }}, \ldots, s_{n}^{\text {min }}\right)
$$

[Łyko, Rudek 2013] can always be found, where

$$
s_{i}^{\min }=\min _{\mathrm{S} \in D P(P, H, m, M)} s_{i} .
$$

The sequence $S^{\min }$ does not have to be an element of the set $D P(P, H, m, M)$. Its terms are numbers indicating the amount of the good available to each entity in compliance with the rules of degressive proportionality.

Lemma. If $S \in D P(P, H, m, M)$ and for all $i \in\{2,3, \ldots, n-1\}$

$$
\frac{p_{i}}{p_{i+1}} \leq 1-\frac{1}{s_{i+1}^{\min }} \text {, then } \frac{p_{i}}{s_{i}-1} \leq \frac{p_{i+1}}{s_{i+1}} \text { and } \frac{p_{i}}{s_{i}} \leq \frac{p_{i+1}}{s_{i+1}+1} \text {. }
$$

Proof. If $\frac{p_{i}}{p_{i+1}} \leq 1-\frac{1}{s_{i+1}^{\min }}$ then

$$
\begin{gathered}
\frac{p_{i}}{p_{i+1}} \leq 1-\frac{1}{s_{i+1}}=\frac{s_{i+1}}{s_{i+1}}-\frac{1}{s_{i+1}} \leq \frac{s_{i}}{s_{i+1}}-\frac{1}{s_{i+1}}=\frac{s_{i}-1}{s_{i+1}} \text { and therefore } \\
\frac{p_{i}}{s_{i}-1} \leq \frac{p_{i+1}}{s_{i+1}} .
\end{gathered}
$$

Moreover for all $0 \leq a \leq b$ and $b \neq 0$ following inequality $\frac{a}{b} \leq \frac{a+1}{b+1}$ holds and hence

$$
\frac{s_{i}-1}{s_{i+1}} \leq \frac{s_{i}-1+1}{s_{i+1}+1} \leq \frac{s_{i}}{s_{i+1}+1} .
$$

It means that $\frac{p_{i}}{p_{i+1}} \leq \frac{s_{i}}{s_{i+1}+1}$ and therefore $\frac{p_{i}}{s_{i}} \leq \frac{p_{i+1}}{s_{i+1}+1}$.

Theorem 1. If the set $D P(P, H, m, M)$ is not empty, if $S$ is not its greatest element under the antilexicographic order, and if for any $i \in\{2,3, \ldots, n-1\}$ the inequality $\frac{p_{i}}{p_{i+1}} \leq 1-\frac{1}{s_{i+1}^{\min }}$ holds, then there exist such numbers

$$
k, l \in\{2,3, \ldots, n-1\} \text { that } S_{k, l}^{+} \in D P(P, H, m, M) .
$$

Proof. Let $S^{*}=\left(s_{1}^{*}, s_{2}^{*}, \ldots, s_{n}^{*}\right)$ be the greatest element of the set $D P(P, H, m, M)$ under the antilexicographic order, and let $l=\max _{i \in\{1,2, \ldots, n\}} s_{i} \neq s_{i}^{*}$. Hence, it is known that $l<n$ and $s_{l}<s_{l}^{*}$. In view of $s_{l}<s_{l}^{*}$ and

$$
s_{1}+s_{2}+\cdots+s_{n}=s_{1}^{*}+s_{2}^{*}+\cdots+s_{n}^{*}=H
$$

we have $s_{j}>s_{j}^{*}$ for a certain $j$. 
Let $k^{\prime}=\max _{i \in\{1,2, \ldots, n\}} s_{i}>s_{i}^{*}$.

We demonstrate that if $k=\min _{i \in\{1,2, \ldots, n\}} s_{i}=s_{k^{\prime}}$, then

$$
S_{k, l}^{+} \in D P(P, H, m, M) \text {. }
$$

Evidently, $k>1$, because of $s_{k}>s_{k}^{*}$. In addition, inequalities $s_{k-1}<s_{k}$ result from the definition of numbers $k$ and $l$, therefore condition (1) in the definition of the set $D P(P, H, m, M)$ is satisfied.

The inequalities $\frac{p_{k}}{s_{k}^{+}} \leq \frac{p_{k+1}}{s_{k+1}^{+}}$and $\frac{p_{l-1}}{s_{l-1}^{+}} \leq \frac{p_{l}}{s_{l}^{+}}$hold by lemma, because if $\frac{p_{i}}{s_{i}-1} \leq \frac{p_{i+1}}{s_{i+1}}$ holds, then in particular $\frac{p_{k}}{s_{k}^{+}}=\frac{p_{k}}{s_{k}-1} \leq \frac{p_{k+1}}{s_{k+1}}=\frac{p_{k+1}}{s_{k+1}^{+}}$is true, while $\frac{p_{l-1}}{s_{l-1}^{+}}=\frac{p_{l-1}}{s_{l-1}} \leq \frac{p_{l}}{s_{l}+1}=\frac{P_{l}}{s_{l}^{+}}$results from $\frac{p_{i}}{s_{i}} \leq \frac{p_{i+1}}{s_{i+1}+1}$.

Theorem 2. If the set $D P(P, H, m, M)$ is not empty and $S$ is not its greatest element under the lexicographic order, and if the inequality $\frac{p_{i}}{p_{i+1}} \leq 1-\frac{1}{s_{i+1}^{\min }}$ holds for any $i \in\{2,3, \ldots, n-1\}$, then there exist such numbers $k, l \in\{2,3, \ldots, n-1\}$ that $S_{k, l}^{-} \in D P(P, H, m, M)$.

Proof. Let ${ }^{*} S=\left({ }^{*} s_{1},{ }^{*} s_{2}, \ldots,{ }^{*} s_{n}\right)$ be the greatest element of the set $D P(P, H, m, M)$ under the lexicographic order, and let $k^{\prime}=\min _{i \in\{1,2, \ldots, n\}} s_{i} \neq{ }^{*} s_{i}$. It follows that $k^{\prime}>1$ and $s_{k^{\prime}}<{ }^{*} s_{k^{\prime}}$.

Because of $s_{1}+s_{2}+\cdots+s_{n}={ }^{*} s_{1}+{ }^{*} s_{2}+\cdots+{ }^{*} s_{n}=H$, there exists such $j$ that $s_{j}>{ }^{*} s_{j}$. Let $l=\min _{i \in\{1,2, \ldots, n\}} s_{i}>{ }^{*} s_{i}$. We demonstrate that $S_{k, l}^{-} \in D P(P, H, m, M)$ holds for $k=\max _{i \in\{1,2, \ldots, n\}} s_{i}=s_{k^{\prime}}$. From the definition of numbers $k$ and $l$ we know that

$$
s_{k}<s_{k+1}, s_{l}>{ }^{*} s_{l} \text { and } s_{l-1} \leq{ }^{*} s_{l-1} \text {, }
$$

thus the condition (1) in the definition of the set $D P(P, H, m, M)$ is satisfied.

As in the proof of Theorem 1, one can observe that inequalities

$$
\begin{gathered}
\frac{p_{k-1}}{s_{k-1}^{-}} \leq \frac{p_{k}}{s_{k}^{-}} \text {and } \frac{p_{l}}{s_{l}^{-}} \leq \frac{p_{l+1}}{s_{l+1}^{-}} \text {are special cases of inequalities } \\
\frac{p_{i}}{s_{i}} \leq \frac{p_{i+1}}{s_{i+1}+1} \text { and } \frac{p_{i}}{s_{i}-1} \leq \frac{p_{i+1}}{s_{i+1}} \text {, respectively, }
\end{gathered}
$$

which implies that $S_{k, l}^{-} \in D P(P, H, m, M)$. 


\section{Conclusions}

Transferring one unit of a good between agents is not always possible so as to meet the rules of degressively proportional allocation. In fact this opportunity depends on the diversification of the set $P$ of claims. The proven theorems imply that a transfer of goods between agents can be accomplished on the condition that $\frac{p_{i}}{p_{i+1}} \leq \frac{m-1}{m}$, because it is the smallest value attainable by the sequence $S^{\min }$. As a result it is possible to control the boundary conditions in such a way that transfers of goods both from smaller as well as from larger agents always take place in the set $D P(P, H, m, M)$. The only limits in this respect are determined by the greatest elements under antilexicographic and lexicographic orders.

\section{Acknowledgements}

The results presented in this paper have been supported by the Polish National Science Centre under grant no. 2013/09/B/HS4/02702.

\section{Bibliography}

Dniestrzański P., Łyko J. (2014). Influence of boundary conditions of digressively proportional division on the potential application of proportional rules. Procedia - Social and Behavioral Sciences. Vol. 109, pp. 722-729.

Delgado-Márquez B., Kaeding M., Palomares A. (2013). A more balanced composition of the European Parliament with degressive proportionality. European Union Politics. No 14, pp. 458-471.

Lamassoure A., Severin A. (2007). European Parliament Resolution on "Proposal to amend the Treaty provisions concerning the composition of the European Parliament”. Adopted on 11 October 2007 (INI/2007/2169).

Łyko J. (2012). The boundary conditions of degressive proportionality. Procedia - Social and Behavioral Sciences 65, pp. 76-82.

Łyko J., Rudek R. (2013). A fast exact algorithm for the allocation of seats for the EU Parliament. Expert Systems with Applications 40 (13), pp. 5284-5291.

Pukelsheim F. (2013). Proportional Representation. Apportionment Methods and Their Applications. Springer.

The Treaty of Lisbon (2007). http://europa.eu/lisbon_treaty/ full_text/index_en. (Accessed: 15.02.2012). 